\title{
Understanding the short-range magnetic correlations in MnTe through magnetic pair distribution function analysis \\ Jacob Christensen ${ }^{1}$, Benjamin Frandsen ${ }^{2}$ \\ ${ }^{1}$ Brigham Young University ${ }^{2}$ Brigham Young University \\ christensenjacob763@gmail.com
}

The antiferromagnetic semiconductor MnTe has recently attracted significant attention as both a high-performance thermoelectric and a candidate material for spintronics. The magnetic properties of MnTe play a crucial role in both of these technological applications. MnTe has a hexagonal layered structure in which magnetic Mn2+ spins order ferromagnetically within the plane and antiferromagnetically between the planes below $\mathrm{TN}=307 \mathrm{~K}$. Above TN, robust short-range magnetic correlations survive to high temperature. It has been shown that these short-range correlations are a significant contributor to the high thermoelectric figure of merit zT in MnTe through a mechanism known as paramagnon drag. Here, we present comprehensive atomic and magnetic pair distribution function (PDF) analysis of neutron total scattering data collected from pure and doped MnTe powders, together with threedimensional magnetic PDF data obtained from a single crystal of MnTe. These complementary data sets allow us to track in detail the evolution of the magnetic correlations from the long-range ordered state at low temperature to the short-range ordered state at high temperature. We present real-space magnetic models that reproduce the observed mPDF patterns with quantitative accuracy and discuss the significance of these results in the context of existing work on MnTe. 